\title{
Research on the Application of Ant Colony Algorithm in Underwater Path Planning
}

\author{
Wei Feng ${ }^{1, a}$, Zhe Rao ${ }^{1, b}$, Zhong Wang ${ }^{1, c}$ \\ ${ }^{1}$ Department of Weaponry Engineering, Naval University of Engineering, Wuhan, 430033, China \\ aemail: 476507122@qq.com, bemail: icesnowaloe@163.com, email:dtoubaby@163.com
}

\begin{abstract}
Keywords: Underwater terrain-aided navigation; Path planning; Ant colony algorithm; Particle filter Abstract. In order to ensure sufficient terrain information for the underwater terrain-aided navigation, a global path planning method based on Ant Colony Algorithm (ACA) is proposed. The concept of feasible region is firstly defined, and then pheromone representation, heuristic functions and pheromone updating rules are discussed in detail. Thirdly, ACA considering comprehensively navigation tasks and terrain information is studied on the basis of real terrain data. Finally, Simulative verification with Particle Filter (PF) navigational algorithm on the given path is conducted. The results show that the proposed path planning method can achieve good performance in underwater terrain-aided navigation.
\end{abstract}

\section{Introduction}

Terrain-aided Navigation (TAN) is a terrain-based technology for aircrafts or underwater vehicles to navigate and position. The working principle of TAN is like this: first pre-input the topographic data (reference map) of certain area into underwater vehicle's computer, and then when it goes through this area, it can measure the topographic information along the path with its sensor (real-time image), applying matching algorithm, we can get simultaneous location of the vehicle [1]. With this regard, to scheme a topographically informative path is critical for good terrain contour matching performance. Path planning should take many factors, such as navigable information, navigation accuracy, digital map error and possible threats, into consideration. The navigability of matching area is generally decided and analyzed with characteristic parameter distribution, because probability of wrong match in amplitude flat areas is much bigger than that in undulated areas. This paper is going to define navigation coefficient of certain area by combining topographic standard deviation and the absolute roughness values [2,3](in warp and weft direction) and also to set the max-min any system algorithm [4] as a basic optimizing search algorithm in studying underwater TAN-based path planning. An underwater topographic condition model will be designed as well as a path planning search algorithm, whose accuracy and efficiency will be proved by simulation experiment result.

\section{Underwater Environment Model}

This paper mainly discusses how to plan an optimal path relying on terrain data in order to achieve better matching results, which has no direct relevance to depth, therefore we will only focus on simplified two-dimensional path planning model.

Usually, submarine topographic data used in TAN adopts Digital Elevation Model (DEM) that is stored in lattice matrix, while dividing terrain plane into average grid. Figure 1(a) is a three-dimensional view of a real digital map simulated in this research and figure 1(b) is its bathymetric contour chart.

To process the map with multilinear polynomial interpolation, we will get a digital map with 20 meters accuracy. We've set the left bottom of the map as origin of coordinates, latitudinal level as $X$-axis (positive east direction), longitudinal $Y$-axis (position north direction) and divided the map with lines respectively parallel to $X, Y$ axis to be a $20 m \times 20 m$ discrete point set. 


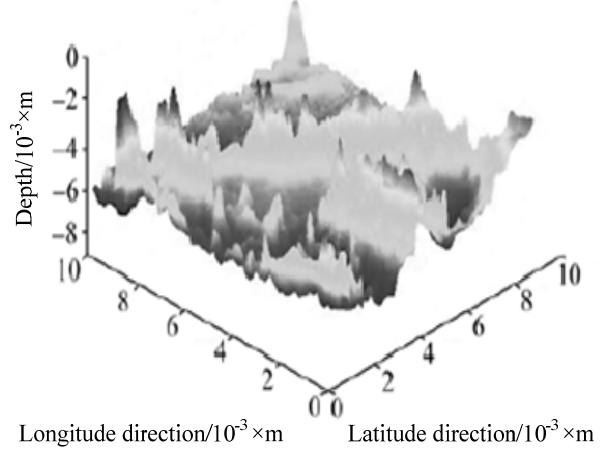

(a) Three-dimensional view of a terrain map

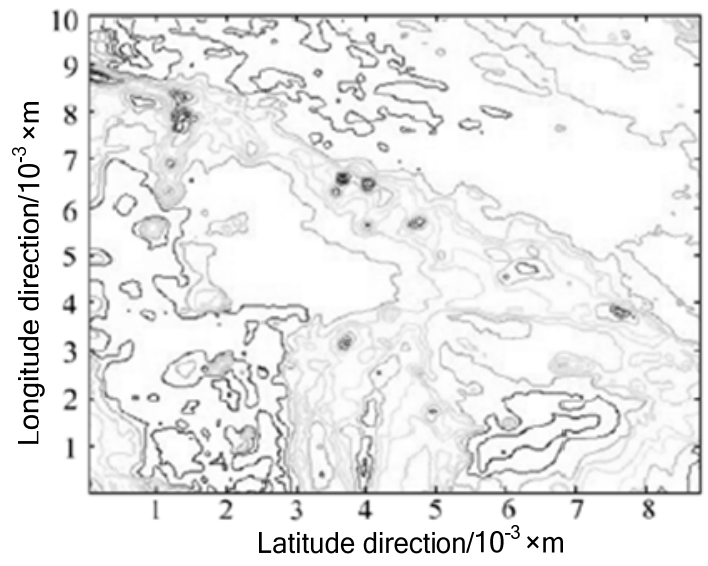

(b) Bathymetric contour chart

Fig.1 Sea-bottom digital map of certain area

\section{Path Planning Algorithm}

Feasible region selection. If we divide the digital map in cartesian grid, searching from current point to the next consecutive point, until the end of the planned path, thus a desirable path connecting the start with the end is able to generate. Fig. 2 is the data structure of trajectory point $O$. The data structure of this grid algorithm is a nine-grid diagram centered on current point. It has 8 consecutive points, from which should the next stop be selected. The side length of every single grid $\left(d_{1}\right)$ should be reasonably decided in accordance to the size of real problem as well as the resolution of digital map. Here set $d_{1}$ as the resolution of digital map while $R$ as the turning radius of the vehicle.

$$
\frac{1}{2} d_{1}<R<\frac{3}{2} d_{1}
$$

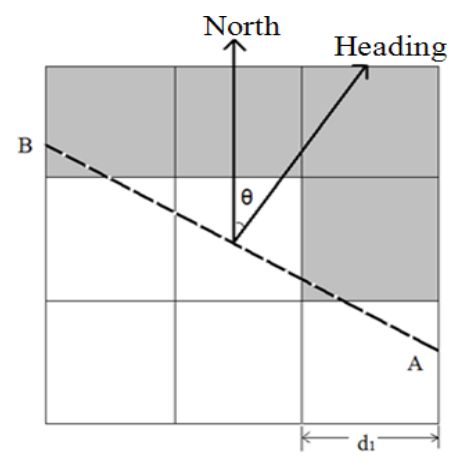

Fig.2 Schematic of feasible region

If the relation of $R$ and $d_{1}$ meets with the above condition, we bring up the conception of feasible region in order to reduce the spatial and temporal complexes in path planning. Say our vehicle is not at point $O$, heading angle $\theta$, line $A B$ perpendicular to heading direction, and then $A B$ has divided this nine-grid diagram into two parts. The one coordinating our heading direction will be the next feasible region $\Psi$ for underwater vehicle. If line $A B$ goes through one single grid, then whether it's feasible grid should be decided according to the nature of its larger part. That is, if more than a half of this grid falls in feasible region, this grid should be included and vice versa, like the dash area illustrated in Fig.2. During path planning, the underwater vehicle is just like ant in Ant Colony Algorithm (ACA). The heading direction of ant is decided by its locations at time $t$ and $t-1$.

Pheromone representation. ACA was first used in dealing with Traveling Salesman Problem (TSP). Since the environment model used to cope with two-dimensional path planning questions in 
this paper is in the form of discrete point set, the points in it exactly correspond to cities in TSP. Thus if we take the connecting lines between each two discrete points as carrier for pheromone, the spatial complicacy of this algorithm will be unimaginable. With this respect, in this research, the pheromone will be stored at every discrete point in the environment model and the size of each pheromone will be read as its degree of attraction to ant. In this way, the spatial complicacy is significantly decreased.

Path nodes selection. The procedure of deciding an ant going from $S_{t}\left(x_{t}, y_{t}\right)$ to next node $S_{t+1}\left(x_{t+1}, y_{t+1}\right)$ is as follows:

a. Decide the feasible region for the ant according to its heading direction, which is decided by its current location $S_{t}\left(x_{t}, y_{t}\right)$ and location of last moment $S_{t-1}\left(x_{t-1}, y_{t-1}\right)$;

b. Calculate the total heuristic function $\eta_{i j}(t)$ of a random grid $j$ from feasible region, and then the heading direction $\theta_{i j}$ from current location $S_{i}\left(x_{i}, y_{i}\right)$ to $S_{j}\left(x_{j}, y_{j}\right)$;

c. Calculate the transition probability of ant $k$ from $S_{i}\left(x_{i}, y_{i}\right)$ to $S_{j}\left(x_{j}, y_{j}\right)$ with function (2):

$$
P_{i j}^{k}(t)=\left\{\begin{array}{l}
\frac{\tau_{i j}^{\alpha}(t) \tau_{i j}^{\beta}(t)}{\sum_{s \in \Psi} \tau_{i s}^{\alpha}(t) \tau_{i s}^{\beta}(t)}, j \in \Psi \\
0 \quad, \text { else }
\end{array}\right.
$$

in which, $\tau_{i j}(t)$ represents the intensity of pheromone at location $S_{j}\left(x_{j}, y_{j}\right)$, time $t$ (i.e.,attractive factor); $\eta_{i j}(t)$ is a heuristic function from $S_{i}\left(x_{i}, y_{i}\right)$ to $S_{j}\left(x_{j}, y_{j}\right)$ at time $t ; \alpha$ and $\beta$ are the influence coefficients to pheromone and heuristic function respectively;

d. Ants transit to next node $S_{j}\left(x_{j}, y_{j}\right)$ according to the pseudo-random proportional rule presented in expression (3);

$$
P_{j}(t)=\left\{\begin{array}{l}
\arg \max _{s \in \Psi}\left[\tau_{i j}^{\alpha}(t) \eta_{i j}^{\beta}(t)\right], q \leq q_{0} \\
S \quad \text { else goto }(2)
\end{array}\right.
$$

in which, $q$ is a uniformly distributed random number between section $[0,1] ; q_{0}$ is parameter ( $\left.0 \leq q_{0} \leq 1\right)$; $S$ is a random variable selected from the probability distribution generated from function (2).

Design of heuristic function.Heuristic function plays a vital role in effectively planning out a desirable navigation path. The path planned in this paper aims at better TAN performance, with regard to which, length of the path and the terrain navigability are emphasized in optimizing the navigation path. Accordingly, our heuristic function $\eta_{i j}(t)$ is like this:

$$
\eta_{i j}(t)=\left[T_{i j}(t)\right]^{\beta_{1}} \times\left[D_{i j}(t)\right]^{\beta_{2}} \times\left[N_{i j}(t)\right]^{\beta_{3}}
$$

in which, $D_{i j}(t)$ is speedy navigation factor (i.e., the shortest path length); $N_{i j}(t)$ is other factors in mission planning, like threatened areas; $T_{i j}(t)$ is topographic information; $\beta_{1} \beta_{3} \beta_{2}$ are weighting coefficients, respectively representing the relative significance of above factors. Their values are decided in terms of requirements in actual planning; $T_{i j}(t)$ is the navigation coefficient of point $S_{j}\left(x_{j}, y_{j}\right)$ in topographic region, and

$$
T_{i j}(t)=\sigma_{i j}+r_{x}^{i j} \sin \theta+r_{y}^{i j} \cos \theta
$$


in which, $\sigma_{i j}, r_{x}^{i j}, r_{y}^{i j}$ are topographic standard deviation and the absolute roughness values in $x$ and $y$ directions of point $S_{j}\left(x_{j}, y_{j}\right)$ respectively.

Pheromone update. To avoid stagnant search, the range of pheromone is confined in section $\left[\tau_{\min }, \tau_{\max }\right]$, and the initial value set as $\tau_{\max }$ to allow the ant able to get new solution from the very beginning. There are partial and overall updates. The purpose of the former is to enable ants to find different solutions and avoid them converging to one single path too soon. Partial update happens during ants' transition. When ant $k$ moves to node $s$, the pheromone at $s$ will update according to equation (6):

$$
\tau_{s}=(1-\rho) \tau_{s}
$$

in which, $\rho$ is the pheromone volatile coefficient. After all the ants have completed one patch construction, overall update follows. Under the principle of shortest path, every completed iteration should be ensued with one pheromone update on temporary optimal path according to equation (7).

$$
\tau_{j}(t+1)=\tau_{j}(t)+\Delta \tau_{j}^{\text {best }} \text {. }
$$

in which, $\Delta \tau_{j}^{\text {best }}=1 / f\left(s^{\text {best }}\right), f\left(s^{\text {best }}\right)$ are the values of the optimal solution $s^{\text {ib }}$ in this iteration.

\section{Steps of algorithm implementation.}

a. Process digital map; use Eq.5 to calculate the value of navigation coefficient in adopted region;initialize parameters and environmental data, give pheromone matrix an initial value; decide the starting and ending points of planned path; put all the ants at the starting point.

b. Decide each ant's next feasible region; calculate the heuristic function values of points in feasible region with Eq.3, and decide ants' next stops with generated function values as well as pheromone values under Eq.2 and Eq.3.

c. When ants transit to the next navigation nodes, update partial pheromone under Eq.6.

d. Repeat steps $\mathbf{b}$ and $\mathbf{c}$ until all the ants have completed one path construction, and then in accordance to optimal navigation path rule $f\left(s^{\text {best }}\right)$, work out temporary iterative optimal path.

e. Conduct overall update under Eq.7 and decide whether the algorithm should stop (the algorithm has already converged or had enough iterations). If so, output the ultimate result; if not, do step $\mathbf{b}$ all over again.

\section{Simulative verification}

To test the validity of above presented ACA, we'll run simulative experiment on it with Matlab 7.0. This test is conducted on the basis of real digital map of Fig. 1 with $20 \mathrm{~m} \times 20 \mathrm{~m}$ grid model. Set the starting coordinates as $(1200,4900)$, and the ending $(8000,3900)$. After several simulative calculations, desirable will be generated if we initialize basic parameters as follows: $\alpha=1.0, \beta_{1}=\beta_{3}=1.0, \beta_{2}=2.0, \rho=0.05$, number of ants $M=20$, iteration times $N=100$.

1) If other factor $N_{i j}(t)$ is excluded, i.e., don't take influential factors like threats into consideration, resulted optimal path from the ACA-based path planning optimization algorithm is marked with solid line in Fig.3.

2) If take $N_{i j}(t)$ into consideration, assuming $N_{i j}(t)$ as threatened area, circled out in simulation, when planning navigation path, we need to avoid such area and the generated optimal path is marked with solid line in Fig.4.

3) In order to test feasibility, set planned path from 1) as underwater vehicle's actual navigation path and write underwater terrain matching program based on Particle Filter (PF) algorithm[5], we'll get a terrain matching path as indicated with double lines in Fig.3, matching errors indicated in Fig.5. If no path planning activity included, that is to set a straight line from the start to the end as the actual navigation path, the matching errors are like Fig.6. From the matching results, the optimal path 
generated with TAN enjoys more rapid convergence, which testifies to its efficiency in terrain matching.

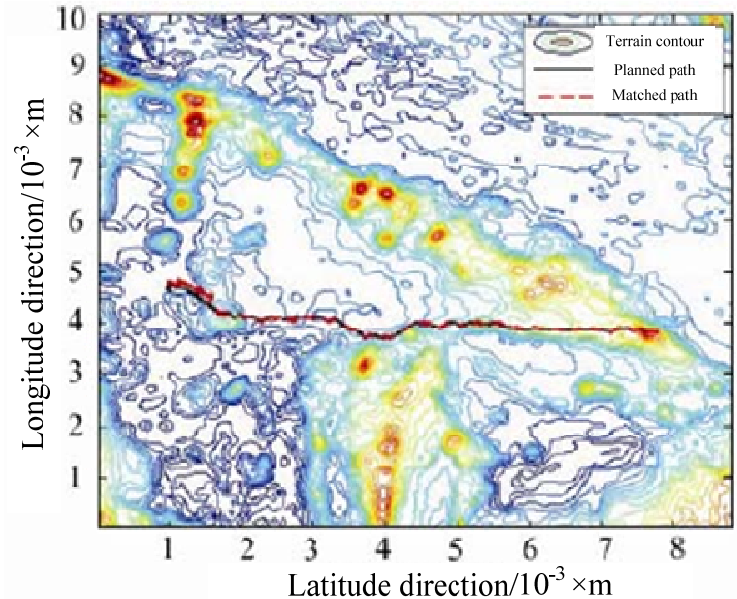

Fig.3 Planed path without regard to threat

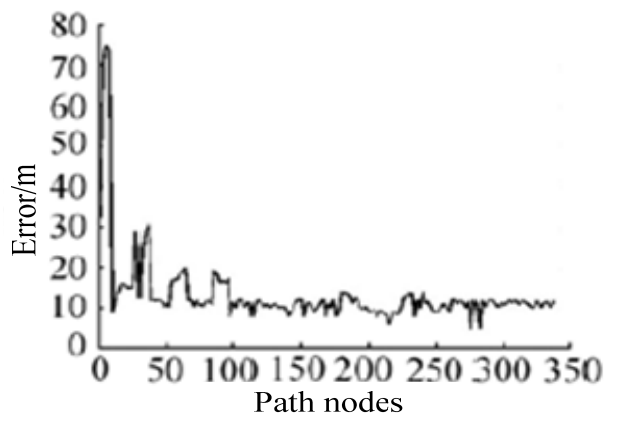

Fig.5 Matching errors of planned path

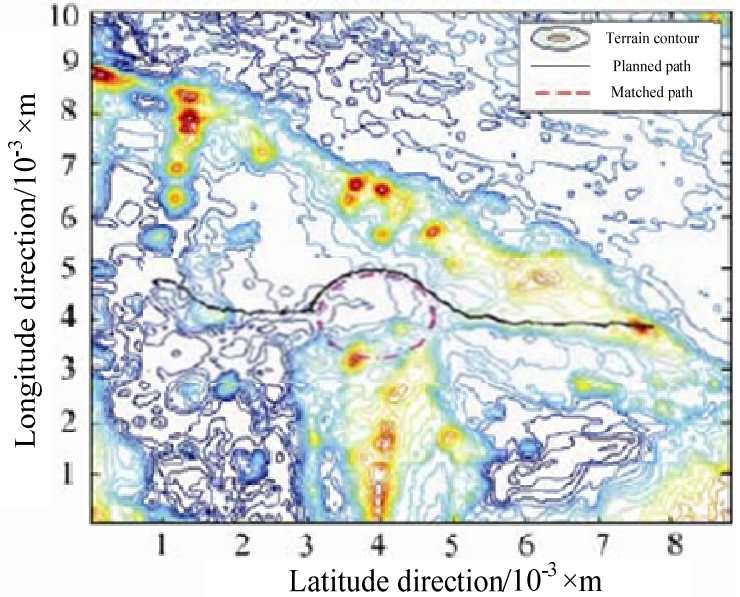

Fig.4 Planed path with regard to threat

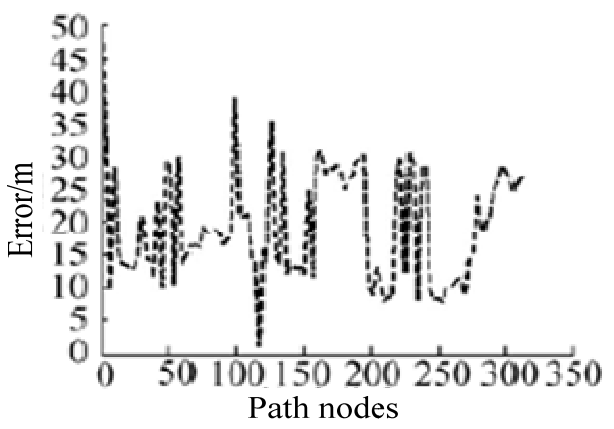

Fig.6 Matching errors of straight path

\section{Conclusion}

In regard to underwater terrain-aided navigation, this paper adopts ant colony optimization algorithm to deal with path planning relevant questions for underwater vehicles. The path optimizing research algorithm has been elaborated with specific implementing procedures and simulative tests on planned optimal path have been run on real digital map under PF algorithm, whose result testifies to the efficiency and accuracy of our designed algorithm in planning optimal navigation path with regard to desirable matching performance in underwater TAN.

\section{Reference}

[1] Nygren I. Terrain Navigation for Underwater Vehicles[D]. Sweden:Royal Institute of Technology (KTH), 2005.

[2] Tong Zheng,Long-fei Cai,Zhi-gang Wang. Selection of Matching Area in Terrain Match Aided Navigation. Journal of China Inertial Technology, 2009, 17(2): 191-196.

[3] Jian Shen,Heng Li,Jing-yuan Zhang. Optimal Path planning Method for Underwater Terrain-aided Navigation[J]. Torpedo Technology,2012,20(4):276-380.

[4] Dorigo M,Thomas S. Ant Colony Optimization[M]. Brussels:MIT,2004:33-45.

[5] Jian Shen,Jing-yuan Zhang,Ping Yan. An Underwater Terrain Matching Arithmetric Based on Particle Filter[J]. Journal of Naval University of Engineering,2008,20(6):107-112. 